\title{
Dynamic Modelling and Analysis of Performance for the Electromagnetic-driven Spherical Robot
}

\author{
Shengju Sang \\ School of Information Science and Technology, Taishan University, Taian Shandong, 271021, China
}

\begin{abstract}
This paper analyses the dynamic performances of the electromagnetic-driven spherical robot including rolling, obstacle overrunning, uphill climbing and the like, as well as discusses the influence factors on the dynamic performance of the spherical robot, which comprise the equivalent pendulum mass, the spherical robot mass and the pendulum length to the spherical robot. Form the above discussions, it becomes obvious that the conclusion can be reached that the larger the ratio defied as $\lambda$, the better of the dynamic performance is. Obviously, a same conclusion can be drawn with regard to the ratio defined as $k$.
\end{abstract}

Keywords-spherical robot; dynamic modeling; electromagnetic driven; obstacle overrunning; uphill climbing.

\section{INTRODUCTION}

Spherical mobile robot, which has a ball-shaped outer shell to accommodate all its mechanism and control devices in it, is characterized as walking mainly in a rolling way, simple, small friction ,compact, well-sealed structure, never turning over and so on. These advantages provide the spherical robots with strong viability and applications in many fields, such as military, transportation, surveillance, search and rescue, toys, entertainment etc. It has attracted the interest of many researchers.

In fact, as early as in 1893, Tate J.L. invented a spherical toy, and applied for a patent [1]. In 1909, Robert E. Cecil designed an amusing spherical toy rolling along a track of zig-zig, and then he applied for a patent [2]. In the subsequent nearly a century, more than 80 patents related to the ball-shaped object were produced. However, few patents came into use, for neither mathematical studies nor experimental researches were carried out until about twenty years ago.

In 1906, Brown H. Benjamin, Jr. and Xu Yangsheng at the Carnegie Mellon University designed a disk-shaped rolling robot named as Gyrover with a single wheel moving forwards and backwards, which is not strictly a spherical robot [3]. The static analysis were developed for the robot, yet neither the dynamic study nor the control method was performed. However, the findings has attracted many scientists from many countries, such as Finland, Canada, Italy, Iran, and Sweden and so on. More and more researchers carried out theoretical and experimental exploration [3-7]. Many recent studies have been performed on the theoretical and experimental exploration, and have made some significant progress in mathematical modelling, mechanism designing and so on, which was carried out by a lot of scientists from many countries all over the world [8$14]$.

So far, the studies concerning the kinematics, dynamics model and control strategy seem insufficient. Thus, a great of further studies are required to consummate the kinematics and dynamics models as well as to perfect control methods for the spherical robot. This paper focus on rolling conditions, uphill climbing, obstacle overrunning of an electromagnetic-driven spherical robot in order to lay the foundation for effective control strategies in the future.

\section{MeChanism OF ELECTROMAGNETIC-DRIVEN SPHERICAL ROBOT}

The present spherical robot is composed of a left hemispherical shell, a right hemispherical shell, a magnetic steel ring and an inner driving mechanism, which is called electromagnetic-driven spherical robot as shown in Figure.1.The left hemispherical shell and the right hemispherical shell form a spherical shell assembled through the fixing screws coaxially with the magnetic steel ring on which the permanent magnetic steel pieces are uniformly disposed. N-electrodes of two adjacent permanent magnetic steel pieces are opposite in direction.

The inner driving mechanism positioned in the spherical shell mainly comprises an inner driving bracket, a main shaft, a flywheel, a steering motor, a motor support, a flywheel shaft, an electricity power supply, a controller, electromagnetic coils and the like. The main shaft, on which the other components are hang up through the inner driving bracket, is fixedly connected with the spherical shell through a couple of bears respectively fixed on inner surface of the left and right shell in opposite direction. The flywheel is hung on the inner driving bracket through a bearing. The steering motor shaft is connected with the flywheel through a coupling in order to drive the lower part of the motor to rotate together with the flywheel shaft vertical to the main shaft and the flywheel. The electromagnetic coils disposed on the bracket are arranged symmetrically relative to the steering motor shaft. 


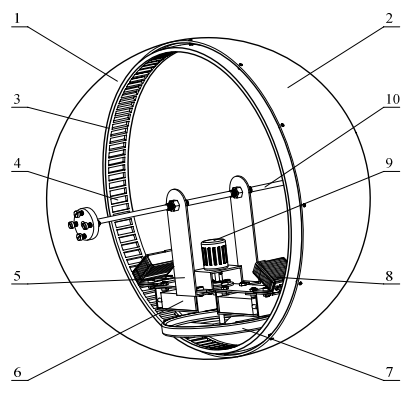

1, left shell, 2, right shell, 3 , steel ring, 4, magnetic steel, 5, bracket6, power and controller, 7, flywheel, 8 , electromagnetic coils, 9 , steering motor, 10 , main shaft

Figure 1. Schematic view of the electromagnetic-driven spherical robot

\section{OBSTACLE OVERRUNNING}

The spherical robot can also overrun an obstacle of certain maximum height. The configuration of a spherical robot encountering an obstacle is shown as Figure. 2.

For simplicity, we have some assumptions as follows,

(1) The spherical robot is in contact with the ground or the obstacles without deformation;

(2) The spherical robot travel in rolling with no slippage;

(3) The initial velocity of spherical robot is zero;

(4) The analysis is based on a two-dimensional plane shown in Figure. 2.

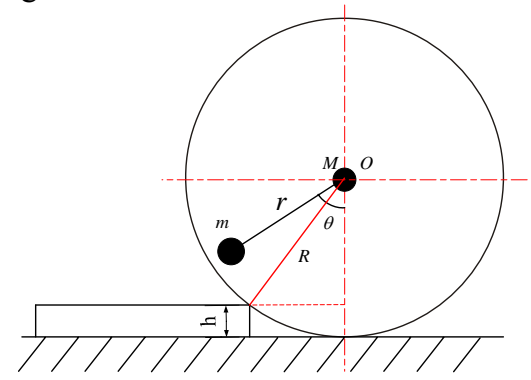

Figure 2. Obstacle over running diagram of the spherical robot

In order to overrun the obstacle, the driving torque must be greater than the counter torque by the gravity. We assume that $\mathrm{h}$ is the height of the obstacle and $\theta$ is the driving angle of the equivalent pendulum. The maximum value of the height of the obstacle can be calculated in the following equations.

$$
m g\left(r \sin \theta-\sqrt{R^{2}-(R-h)^{2}}\right)=M g \sqrt{R^{2}-(R-h)^{2}}
$$

Where, $\mathrm{m}$ is the mass of the equivalent pendulum, $\mathrm{M}$ is the mass of the shell of the spherical robot, $g$ is the gravitational acceleration, $\mathrm{R}$ is the radius of the sphere, and $r$ is the distance between the centers of equivalent pendulum and the spherical shell.

According the Eq. (1), we have,

$$
\begin{aligned}
& h=R-\sqrt{R^{2}-(m r \sin \theta /(M+m))^{2}} \\
& =R\left(1-\sqrt{1-(m r \sin \theta /(M+m) R)^{2}}\right)
\end{aligned}
$$

Assuming that $\mathrm{k}$ is the ratio of $\mathrm{r}$ to $\mathrm{R}$, that is
Hence,

$$
\kappa=r / R
$$

$$
h=R\left(1-\sqrt{1-(\sin \theta)^{2} \lambda^{2} \kappa^{2}}\right)
$$

The fact revealed here is that without slippage, the bigger the driving angle of the equivalent pendulum, the larger the height of the obstacle overrun for the spherical robot would be.

Considering $\theta$ can be close to $\pi / 2$, that is $\sin \theta=1$.Thus, Eq. (3) can be written as

$$
h_{\max } \leq R\left(1-\sqrt{1-\lambda^{2} \kappa^{2}}\right)
$$

Eq. (5) shows that the overrunning ability of the spherical robot depend mainly on the two factors without considering slippage, one is the ratio of $r$ to $R$, and another is the ratio of $\mathrm{m}$ to $(\mathrm{m}+\mathrm{M})$.

(1) The bigger the ratio of $r$ to $R$ is, the larger the height of the obstacle overrun with no slippage for the spherical robot would be. If $r$ can be made close to $\mathrm{R}, h_{\max }$ can be expressed as

$$
h_{\max } \leq R\left(1-\sqrt{1-\lambda^{2}}\right)=R\left(1-\sqrt{1-(m /(m+M))^{2}}\right)
$$

(2) The bigger the ratio of $m$ to $(m+M)$ is, the larger the height of the obstacle would be. If $M$ can be close to 0 compared with $\mathrm{m}, h_{\max }$ will be only influenced by the ratio of $r$ to $R$, that is,

$$
h_{\max } \leq R\left(1-\sqrt{1-\kappa^{2}}\right)=R\left(1-\sqrt{1-(r / R)^{2}}\right)
$$

\section{UPHILL CLIMBING}

The robot is capable to roll uphill in certain inclination as shown in Figure. 3.

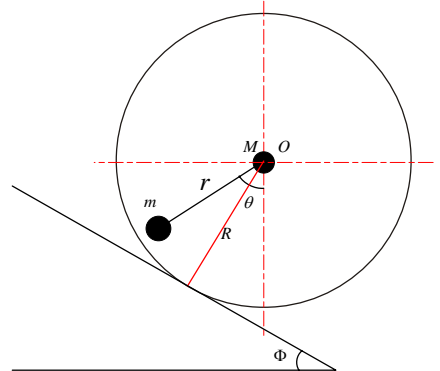

Figure 3. Uphill motion diagram of the spheical robot

The equation for uphill climbing of the spherical robot is given as follows:

$$
m g(r \sin \theta-R \sin \Phi)=M g R \sin \Phi
$$

Where,

M: the mass of the robot,

$\mathrm{m}$ : the mass of the equivalent pendulum,

$\mathrm{R}$ : the radius of the robot,

$\mathrm{r}$ : the distance between centers of the robot and the pendulum, 
$\mathrm{g}:$ the gravitational acceleration,

$\theta$ : the rotation angle of the equivalent pendulum with respect to the robot

$\Phi$ : the slope angle of the inclination.

Assuming the robot rolls without slipping, the slope angle of the inclination can be expressed as

$$
\begin{aligned}
& \Phi=\sin ^{-1}((m \times r) /((M+m) \times R)) \\
& =\sin ^{-1}(\sin \theta \times m /(M+m) \times(r / R))
\end{aligned}
$$

As a consequence of the driving angle of the equivalent pendulum increasing, the inclination slope angle which the robot is cable of climbing would be greatened proportionately.

Considering $\theta$ can be close to $\pi / 2$, that is $\sin \theta=1$, the maximum slope angle of the inclination $\Phi_{\max }$ can be written as

$$
\Phi_{\max }=\sin ^{-1}(m r /(M+m) R)=\sin ^{-1}(m /(M+m) \llbracket r / R)
$$

Hence,

$$
\Phi_{\text {max }}=\sin ^{-1}(\lambda \sqcap \kappa)
$$

Where $\lambda=m /(M+m), \kappa=r / R$.

Note that if $\mathrm{r}$ can be made close to $\mathrm{R}$ or $\lambda$ can be made close to $1, \Phi$ will increase considerably. It seems that the situation becomesunrealistic.

Eq. (11) demonstrates that the inclination slope angle which the robot is cable of climbing is mainly conditioned by two factors, which comprise the ratio of $\mathrm{r}$ to $\mathrm{R}$ and the ratio of $\mathrm{m}$ to $(\mathrm{m}+\mathrm{M})$. A short discussions are given as bellow.

(1) The bigger the ratio of $r$ to $R$, the larger the inclination slope angle for the spherical robot would be. If $r$ can be made close to R, $\Phi_{\max }$ can be rewritten as

$$
\Phi_{\text {max }} \leq \sin ^{-1}(\lambda)=\sin ^{-1}(m /(m+M))
$$

(2) The bigger the ratio of $m$ to $(m+M)$, the larger the inclination slope angle for the spherical robot. If $\mathrm{M}$ can be close to 0 compared with $\mathrm{m}, \Phi_{\max }$ will be only influenced by the ratio of $r$ to $R$, thus, the equation of the maximum of the inclination slope angle becomes,

$$
\Phi_{\max } \leq \sin ^{-1}(\kappa)=\sin ^{-1}(r / R)
$$

Taking the ratios including $r$ to $R$ and $m$ to $(m+M)$ as the horizontal coordinate axes, $h_{\max }$ and $\Phi_{\max }$ as the vertical coordinate axes respectively, A diagram can be obtained, which as shown in Figure. 4.

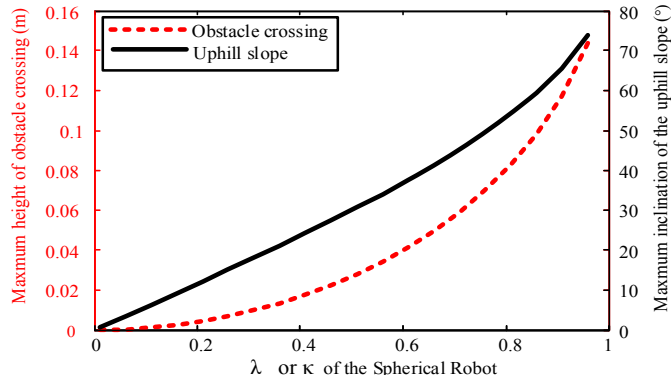

Figure 4. Maximum height of obstacle and inclination angle of slope

A most important fact revealed in Eq. (5) and Eq. (11) is that the variable of $\lambda$ has the same behavior influence with $\kappa$ on $h_{\max }$ and $\Phi_{\max }$, so the variable of $\lambda$ marked in the horizontal coordinate axis are consistent with $\kappa$. As shown in Fig. 6, the red dashed line represented the teensy of $\lambda$ or $\kappa$ versus the maximum height $h_{\max }$, while the black thin lines indicated teensy of $\lambda$ or $\kappa$ versus the maximum slope angle of the inclination $\Phi_{\max }$ respectively. Therefore, it can be concluded that theelectromagnetic-driven spherical robot have the better performance with the increase of the ratios including the equivalent pendulum mass to the spherical robot mass and the length of the pendulum to the spherical robot radius.

\section{CONCLUSIONS}

Based on an electromagnetic-driven spherical robot, we focused on analyses of the dynamic abilities including rolling, uphill climbing, obstacle overrunning and the like in this paper. It follows from what has been said that under the consistent environmental conditions such as materials, rolling friction, sliding friction, etc., the dynamic performance of the spherical robot are mainly conditioned by two factors: one is the radio of the equivalent pendulum mass to the spherical robot mass defined as $\lambda$ and another is the radio of the length of the pendulum to the spherical robot radius defined as $\mathrm{k}$. In conclusion, we can say that hat the electromagnetic-driven spherical robot have the better performance with the increase of the ratios including the equivalent pendulum mass to the spherical robot mass and the length of the pendulum to the spherical robot radius.

\section{ACKNOWLEDGEMENTS}

The Research was partially supported by the Natural Science Foundation of China(61303022), the Natural Science Foundation of Shandong, China(2009AA01105), the science and technology development fund of Tai'an (20133011), and the Foundation provided by Taishan University (Y-01-2013010). The author gratefully acknowledge Professor SHEN Ding for discussion and suggestions, also thank the associate editor and the anonymous reviewers for their valuable comments and kind suggestion to improve the quality of this paper. 


\section{REFERENCES}

[1] J.L., Tate. Toy [P]. USPTO: 508558, 1893-11-14.

[2] Robert E. Cecil. Mechanical toy [P]. USPTO: 933623, 1909-9-7.

[3] Brown H. Benjamin, Jr. and Xu Yangsheng. A single wheel gyroscopically stabilized robot. Proc. IEEE Int. Conf. on Robotics and Automation, 1996(4): 3658-3663.

[4] Aame Halme, Jussi Suomela, etc. Motion control of spherical mobile robot.4th IEEE International Workshop on Advanced Motion Control AMC'96, Mie University, Japan 1996

[5] Das T., and Mukherjee R. Reconfiguration of a rolling sphere: a problem in evolute-involute geometry. Transactions of the ASME Journal of Applied Mechanics, 2006, 73(44): 590-597.

[6] F. Tomik, S. Nudehi, L. L. Flynn, and R. Mukherjee. Design, Fabrication and Control of Spherobot: A Spherical Mobile Robot. J Intell Robot Syst., 2012, 67:117-131.

[7] Bicchi, A., Balluchi, A., Prattichizzo, D., Gorelli,A. Introducing the 'SPHERICLE': An experimental testbed for research and teaching in nonholonom. Proc. IEEE Int. Conf. on Robotics and Automation, 1997, 3:2620-2625.

[8] Michaud F. and Caron S. Roball an autonomous toy rolling robot. In Proc. of the Workshop on Interactive Robot Entertainment(WIRE), CMU Robotics Institute and AAAI, Pittsburgh Pennsylvania, 2000:127-134.
[9] Kang Hou, Hanxu Sun, Qingxuan Jia and Yanheng Zhang. An Autonomous Positioning and Navigation System for Spherical Mobile Robot. Procedia Engineering, 2012, 29:2556-2561

[10] Shengju SANG, Ding SHEN, Jichao ZHAO, Jiayang HU, and Qi AN. Analysis and Simulation of a Spherical Robot. Advanced Materials Research. Vol. 171-172, 2011: 748-751.

[11] Tuanjie Li,Zuowei Wang,and ZhifeiJi.Dynamic Modeling Simulation of the Internal and externa-driven spherical robot. Joural of Aerospace engineering, 2012,10:626-641.

[12] SANG Shengju, ZHAO Jichao, WU Hao, CHEN Shoujun, and AN Qi. Modeling and Simulation of a Spherical Mobile Robot. ComSIS Vol. 7, No. 1, Special Issue, February 2010: 51-62

[13] Yao Cai, Qiang Zhan and Caixia Yan. Two-state trajectory tracking control of a spherical robot using neurodynamics. Robotica, 2012,30:195-203.

[14] Zhao Bo, Wang Pengfei,Hu Haiyan. Design of a spherical robot based on novel double ballast masses principle. High Technology Letter. 2011, 17(2):180-185.

[15] Shengju SANG. Study on Some Driving Mechanisms and Control Strategies for Spherical Robots. Doctor's thesis, East China University of Science and Technology, 2011. 